\title{
Research on Design Method of Structure Parameters of Parallel Robot Driven by Double Sliders Linear Motor
}

\author{
Qingge Kang ${ }^{1,2}$, Ziqiang Zhang ${ }^{1,2, ~ a, ~ R u n d i a n ~} \mathrm{Li}^{1,2}$, Yongjie Zhao ${ }^{1,2}$, \\ Nengsheng Bao ${ }^{1,2}$, Lingsheng Zeng ${ }^{1,2}$ \\ ${ }^{1}$ Key Laboratory of Intelligent Manufacture Technology of Ministry of Education, Shantou \\ University, Shantou, Guangdong, 515063, China \\ ${ }^{2}$ Shantou Institute for Light Industrial Equipment Research, Shantou, Guangdong, 515021, China \\ azqzhang@stu.edu.cn
}

Keywords: Parallel robots, Workspace, Structure parameters, Design method

Abstract. The research actuality and development of parallel manipulators are talked over in this paper. A parallel robot driven by double sliders linear motor has been presented. The kinematics and singularities of the 2 degrees of freedom (DoFs) parallel mechanism of the robot are analyzed. On the basis of that, a new kind of parameters design method based on singularity, workspace and extreme position angle of the mechanism is presented. And the relationships between the structure parameters and the size of reachable workspace, as well as the area of effective workspace based on the method are discussed. The results will provide theoretical basis for application and structure parameters design of the robot.

\section{Introduction}

At present, according to the different forms of the robot structure, the industrial robot can be divided into two categories, serial robots and parallel robots. Compared with the traditional serial robots, the main shortcoming of parallel robots is small workspace. Hence, for the different size of parameters of the robot mechanism, it is the premise of supsequent analysis, synthesis and trajectory planning to grasp the shape and size of workspace.

The workspace is not only an index to evaluate the working region of a mechanism, but also a reference region to define a global index. Normally, to economize the occupied space, we require the workspace/volume ratio of a designed machine to be as large as possible [1].

Generally, robots do not need the whole 6-DoFs to complete tasks in industrial production. Therefore, parallel manipulators with less than 6-DoFs, especially 2 or 3-DoFs have been increasingly attracted attention. Parallel manipulators with 2 or 3-DoFs play an important role in industry and can be applied in parallel kinematics machines, sort, pick and place applications, and other fields. Meanwhile, the parallel robots have the advantages of quick operating frequency, high positioning accuracy, small moving inertia, simple solution of inverse kinematics, easy real-time control etc. Therefore, the parallel robots are easier to realize high speed and precision motion positioning.

The five-bar mechanism is such a typical parallel mechanism with the minimal degrees of freedom (DoFs) in the field, which can be used for positioning a point on a region of a plane that is known as the workspace[2]. Above all, the kinematic design methodology is one of the key parts of kinematic design theory for parallel mechanisms. A relationship between the global conditioning index and the link lengths of two dof planar parallel manipulators was obtained based on a physical model for the solution space [3]. A hybrid method for the optimum kinematic design of two-degree-of-freedom (2-DoF) parallel manipulators with mirror symmetrical geometry was presented [4].

A parallel robot driven by double sliders linear motor has been presented [5]. Parallel mechanism driven by double sliders linear motor, on the one hand, it reduces the complexity of parallel mechanism, which made the whole body more compact and easier to install; on the other hand, it improves precision, sensitivity, rapidity and mobility of the mechanism. In the aspect of the robot structure 
parameters design, a new kind of parameters design method based on singularity, workspace and extreme position angle of the mechanism has been presented.

\section{Structure Description}

The parallel robot driven by double sliders linear motor is shown in Fig. 1. The parallel mechanism is drove by double sliders linear motor 1 , which mainly includes two sliders 12,13 , a stator 11 . Double sliders can slide along the stator. The linear motor is installed on the frame 9. And two sliders are connected to the moving platform 4 by two kinematic chains. The rigidity of the parallel mechanism can be improved effectively by the chain's virtual constraint. Adaptable interface 5 is set on the moving platform, hence different grippers can be installed according to the actual requires [6]. The moving platform's position can be changed within a certain range by changing the displacement of sliders. Each chain is built as a parallelogram mechanism, so the moving platform is always parallel to the fixed platform by the properties of the parallelogram. Therefore, the motion of moving platform is purely translational motion in a plane.

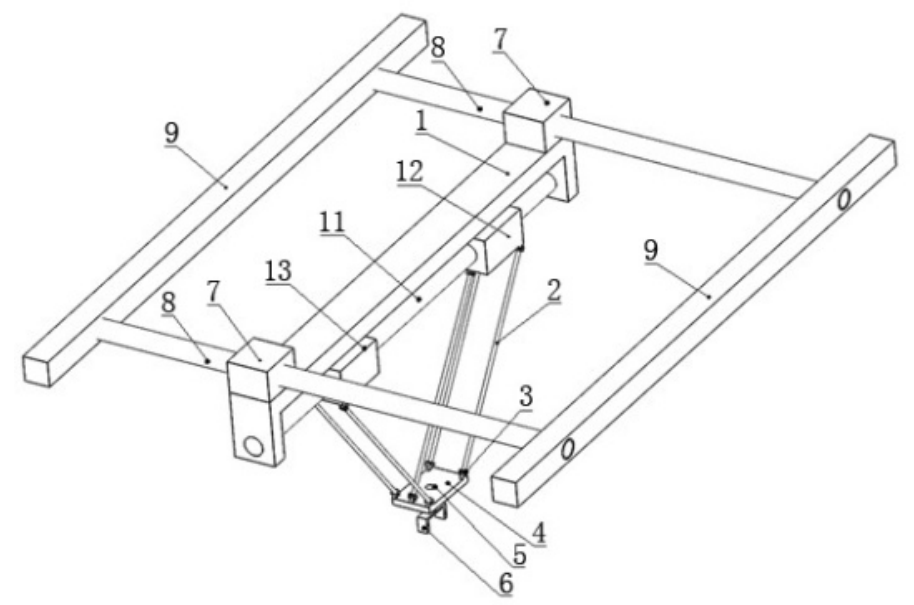

Fig.1 Parallel robot driven by double sliders linear motor

1. Double sliders linear motor, 11.Stator, 12.Left sliders, 13.Right sliders, 2.Rod, 3.Hinge, 4.Moving platform, 5.Adaptable interface, 6.Griper, 7.Sliders, 8.Guide rail, 9.Frame

\section{Kinematics Analysis}

Since the motions of two links of each kinematic chain are identical due to the parallelogram structure, the mechanism kinematic model can be simplified as illustrated in Fig.2. Taking left endpoint of the double sliders linear motor rail as the origin, to establish $O-X-Y$ coordinate system. The base coordinate system $O-X-Y$ is attached to the base with its $X$ axis horizontal through the guide rail. $O, N$ are two endpoints of the guide rail, $O N=L$. The length of each $\operatorname{rod}$ is $R . A, B$ indicate the left and right slider of linear motor respectively. Setting displacement $x_{1}, x_{2}$ of the two sliders as input variable of the mechanism. $O^{\prime}$ is the center point of the moving platform, its $x, y$ displacement as the mechanism's output variable. The angle between the rod and guide rail is $\alpha$. Then, the coordinates of each point in the fixed coordinate system are $O(0,0), N(L, 0), A\left(x_{1}, 0\right), B\left(x_{2}, 0\right), O^{\prime}(x, y)$.

The constraint equation $\left\|A O^{\prime}\right\|=R,\left\|B O^{\prime}\right\|=R$ can be expressed as

$$
\left\{\begin{array}{l}
\left(x-x_{1}\right)^{2}+y^{2}=R^{2} \\
\left(x-x_{2}\right)^{2}+y^{2}=R^{2}
\end{array}\right.
$$




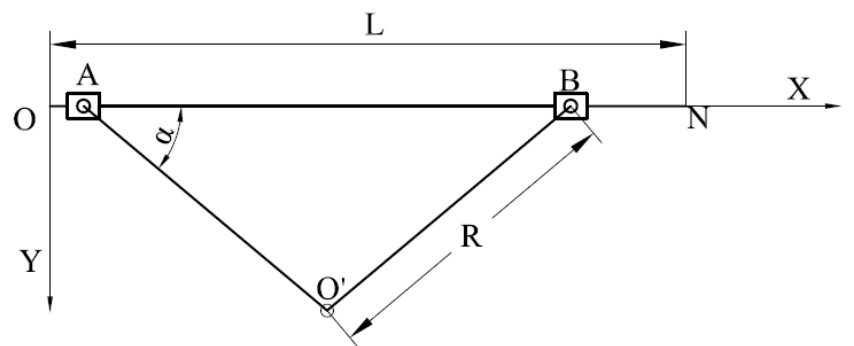

Fig.2 Simplified kinematic model

From Eq. 1, the inverse solutions of the kinematics are

$\left\{\begin{array}{l}x_{1}=x \pm \sqrt{R^{2}-y^{2}} \\ x_{2}=x \pm \sqrt{R^{2}-y^{2}}\end{array}\right.$

For the configuration shown in Fig. 2, the inverse solutions of the kinematics only are

$\left\{\begin{array}{l}x_{1}=x-\sqrt{R^{2}-y^{2}} \\ x_{2}=x+\sqrt{R^{2}-y^{2}}\end{array}\right.$

Taking the derivatives of Eq. 1 with respect to time gives

$\left[\begin{array}{l}x^{\prime} \\ y^{\prime}\end{array}\right]=J\left[\begin{array}{l}x_{1}^{\prime} \\ x_{2}^{\prime}\end{array}\right]$

where $J$ is the Jacobian expressed as

$$
J=\left[\begin{array}{cc}
0.5 & 0.5 \\
0.5 \sqrt{R^{2}-y^{2}} / y & -0.5 \sqrt{R^{2}-y^{2}} / y
\end{array}\right]
$$

While,

$$
\cot \alpha=\sqrt{R^{2}-y^{2}} / y=\cos \alpha / \sin \alpha
$$

so $J$ can be expressed as

$$
J=\left[\begin{array}{cc}
0.5 & 0.5 \\
0.5 \cos \alpha / \sin \alpha & -0.5 \cos \alpha / \sin \alpha
\end{array}\right]
$$

Determinant of $J$ is

$$
|J|=\left|\left[\begin{array}{cc}
0.5 & 0.5 \\
0.5 \cos \alpha / \sin \alpha & -0.5 \cos \alpha / \sin \alpha
\end{array}\right]\right|
$$

then, Eq. 8 can be expressed as

$|J|=-0.5 \cos \alpha / \sin \alpha$

From $|J|=0$, get $\alpha=0$ or $\pi / 2$

Hence, two singular position of the mechanism can be got: the two bars are perpendicular to the guide rail and straightened. Singularities will lead to a loss of controllability and degradation of the natural stiffness of the manipulators, which they must be avoided in the process of the robot structure parameters design.

\section{Workspace Analysis}

Workspace refers to the regional scope which the endpoint of robot can reach. Workspace can be divided into the reachable workspace, dexterity workspace etc. The workspace for the 2-DoFs planar parallel manipulator is a region of the plane derived by the workspace of the reference point $O^{\prime}$ of the moving platform. 
From Eq.1, the reachable workspace of the reference point $O^{\prime}$ is the intersection of the sub-workspaces associated with the two kinematic chains as shown in Fig.3 and 4.

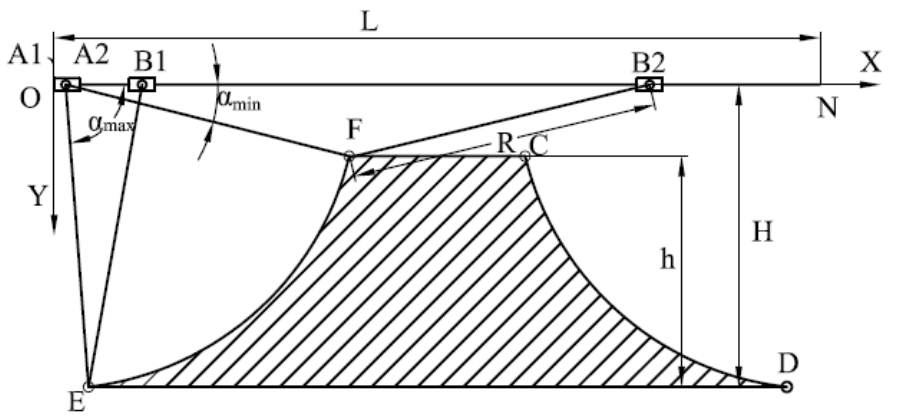

Fig.3 Reachable workspace of the 2-DoFs parallel robot

When $R<L / 2$, the reachable workspace of the parallel robot is shown in Fig. 3. When $R>L / 2$, the reachable workspace of the parallel robot is shown in Fig.4. As it can be seen from Fig.4, the reference point $O^{\prime}$ is faraway from the frame and the effective workspace domain is relatively small and narrow, which is unfavorable for the actual production application. Therefore, this kind of situation should be avoided when determining the parameters of mechanism.

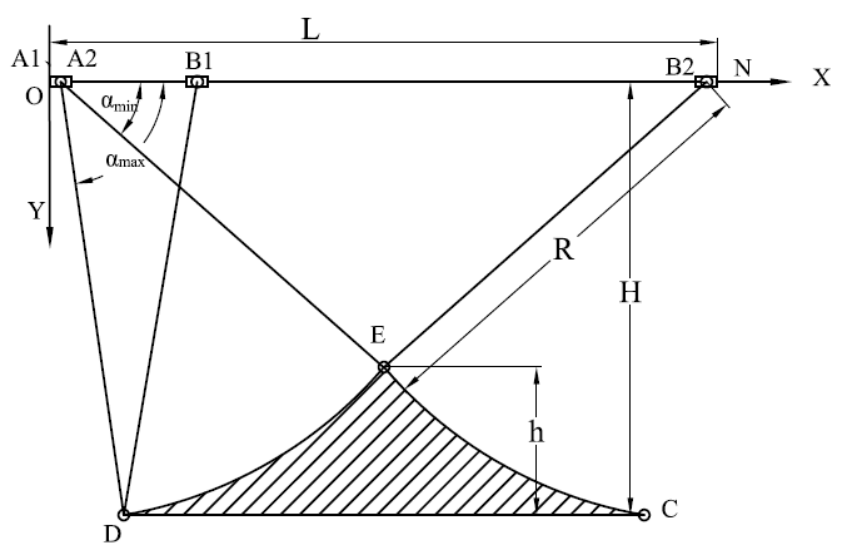

F i g. 4 Reachable workspace of the 2-DoFs parallel robot

\section{Design Method of Mechanism Parameters}

When the size of mechanism is determined, the size of its workspace is certain. There is one to one corresponding relationship between structure parameters and workspace. On the basis of the relationship, the size type with the largest workspace in the structural parameters can be found. Generally speaking, the structural parameters can be changed from zero to infinity, how to use mathematical model to describe the relationship between structure parameters and workspace will be very meaningful.

Constraint Conditions. In order to ensure the force transmission characteristics of the mechanism and avoid singular position, it is necessary to limit the angle between the rod and guide rail. That is

$$
\left\{\begin{array}{l}
\alpha_{\text {min }} \geq[\alpha]_{\text {min }} \\
\alpha_{\text {max }} \leq[\alpha]_{\text {max }}
\end{array}\right.
$$

From Eq.10, the minimum of the angles $\alpha_{\min }$ must be larger than the allowed minimum of the angles $[\alpha]_{\min }$. According to the engineering practice $[\alpha]_{\min }=20^{\circ} \square 25^{\circ}$ is quite reasonable, set $[\alpha]_{\min }=25^{\circ}$ in this mechanism. Actually, both of the sliders have certain size, when the distance of the two sliders is minimum, the angle between the rod and guide rail is maximum. If $\alpha=90^{\circ}$, the 
mechanism would be in the singular position. Generally, $\alpha_{\max }=80^{\circ} \square 85^{\circ}$ is quite reasonable, set $[\alpha]_{\max }=80^{\circ}$ in this mechanism.

Relationship between Reachable Workspace and Structure Parameters. From Fig.3, the following geometry relationships can be got:

$$
\left\{\begin{array}{c}
2 R \cos \alpha_{\min }<L \\
h=R \sin \alpha_{\text {max }}-R \sin \alpha_{\min } \\
C F=L-2 R \cos \alpha_{\min } \\
D E=L-2 R \cos \alpha_{\max }
\end{array}\right.
$$

The relationship between reachable workspace and structure parameters can be expressed as

$$
S=\left(L-R \cos \alpha_{\text {min }}-R \cos \alpha_{\max }\right)\left(\sin \alpha_{\max }-\sin \alpha_{\min }\right) R-2\left(\alpha_{\max }-\alpha_{\text {min }}\right) R^{2}+\sin \left(\alpha_{\max }-\alpha_{\text {min }}\right) R^{2}(12)
$$

where $S$ is the area of reachable workspace.

Take $\alpha_{\text {min }}=25^{\circ}, \alpha_{\text {max }}=80^{\circ}$ into Eq. 12 gives

$S=(0.56 L-0.38 R) R$

From Eq.13, when $R=0.74 L$, get the maximum of $S$.

But by the constraint conditions, $2 R \cos \alpha_{\min }<L, R<L / 2$, when $L \approx 2.1 R$, the larger reachable workspace can be got.

Hence, considering the force transmission characteristics of the mechanism and singular position, when $L \approx 2.1 R$, the larger reachable workspace can be got. It is an important conclusion of this paper.

Performance Index of Workspace. In the process of mechanism design, the actual tasks should be operated in a particular shape region, so the reachable workspace is not necessarily representative of a larger particular shape area. In practical applications, the task workspace is usually defined as a rectangular area in the reachable workspace to evaluate the performance of the robot.

The maximum inscribed quadrilateral is established based on the analysis of the shape of reachable workspace showed in Fig.5.

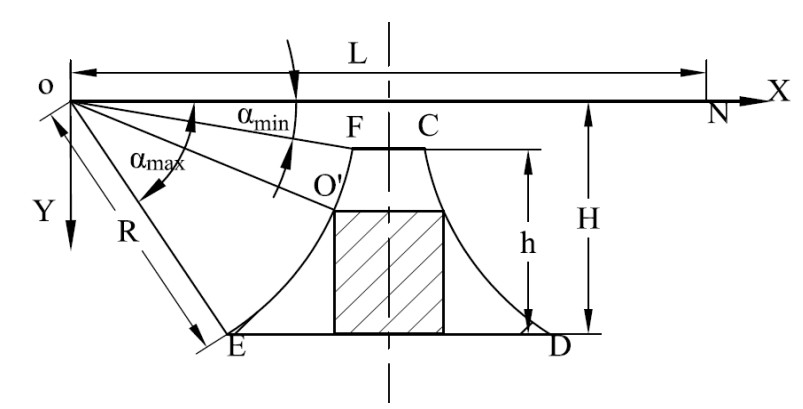

Fig.5 The diagram of maximum inscribed quadrilateral

Let $O^{\prime}=(x, y)$, the area of inscribed quadrilateral can be expressed as

$$
S^{\prime}=2(L / 2-x)\left(R \sin \alpha_{\max }-y\right)
$$

The area satisfies the following constraint conditions:

$$
\left\{\begin{array}{c}
R \cos \alpha_{\max } \leq x \leq R \cos \alpha_{\min } \\
R \sin \alpha_{\min } \leq y \leq R \sin \alpha_{\max } \\
x^{2}+y^{2}=R
\end{array}\right.
$$

where, $\alpha_{\text {max }}=80^{\circ}, \alpha_{\text {min }}=25^{\circ}, L \approx 2.1 R$.

The maximum of $S^{\prime}$ can be calculated by the software of Matlab, that is $S_{\max }^{\prime}=0.19 R^{2}$.

Effective workspace can better reflect the actual work area. From the relationship between effective workspace and structure parameters, if the structure parameters are constant, the actual work area is determined. Conversely, if the certain types of actual operation area are known, the structure 
parameters of mechanism can be selected. The design procedure of structure parameters of parallel robot driven by double sliders linear motor is shown in Fig. 6.

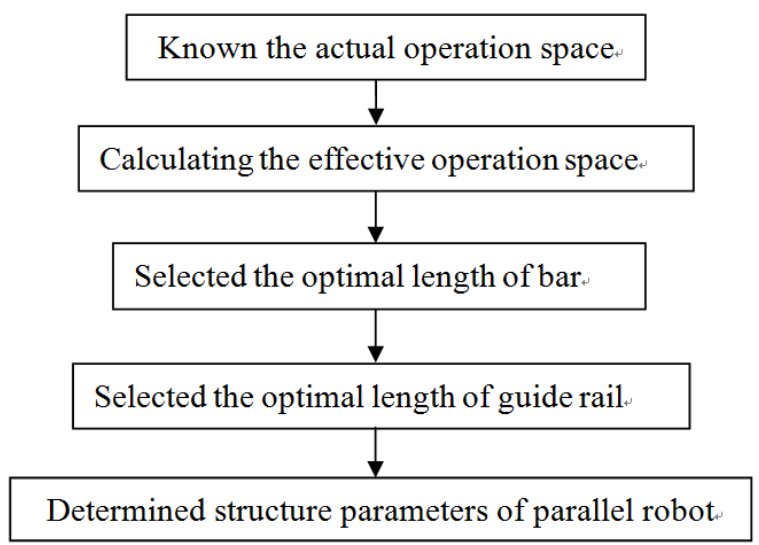

Fig. 6 The design procedure of structure parameters

\section{Conclusions}

A parallel robot driven by double sliders linear motor has been presented. The kinematic and singularities of the 2-DoFs parallel mechanism of the robot has been investigated. $\alpha=0$ or $\pi / 2$ are two singular position of the mechanism. A new kind of mechanism parameters design method based on singularity, workspace and extreme position angle of the mechanism has been presented. And the relationships between the structure parameters and the size of robot mechanism, as well as the area of effective workspace based on the method has been discussed. When $L \approx 2.1 R$, the larger reachable workspace can be got, and the maximum area of effective workspace is $0.19 R^{2}$. The results will provide theoretical basis for the application and structure parameters design of the robot.

\section{Acknowledgements}

The authors wish to thank the Natural Science Foundation of P.R. China for providing a grant (No: 51275281), the Leading Talent Project of Guangdong Province, P.R. China for providing a grant, the Education Department of Guangdong Province, P.R. China for providing a grant (No: 2013CXZDC009) and Guangdong Science and Technology Department, P.R. China for providing grants (No: 2013B011301015 and 2013B011304002) for this project.

\section{References}

[1] X.J. Liu, J.S. Wang and G. Pritschow: Mechanism and Machine Theory, Vol. 41 (2006), No.9, pp.1111-1130.

[2] X.J. Liu, J.S. Wang and G. Pritschow: Mechanism and Machine Theory, Vol. 41 (2006), No.2, pp.145-169.

[3] F. Gao, X.J. Liu and W.A. Gruver: Systems, Man and Cybernetics, 1995. Intelligent Systems for the 21 st Century, IEEE International Conference (Vancouver, Canada, October 22-25, 1995). Vol. 5, pp. 4055 - 4058.

[4] T. Huang, M. Li, Z.X. Li, D.G. Chetwynd and D. J. Whitehouse: Transactions on Robotics and Automation, Vol. 20(2004), No.3, pp.538-543.

[5] Q.G. Kang, Z.Q. Zhang, R.D. Li, Y.J. Zhao and L.S. Zeng: China patent 201410484440.9 (2014). (In Chinese)

[6] L.S. Zeng, Z.Q. Zhang, R.D. Li, Z.W. Zhang and Q.G. Kang: China patent 201420415973.7 (2014). (In Chinese) 